09

\title{
Влияние металлических наночастиц на распространение предельно коротких оптических импульсов в графене
}

\author{
(ㄱ Н.Н. Конобеева ${ }^{1}$, Д.С. Скворцов ${ }^{1}$, М.Б. Белоненко ${ }^{1,2}$ \\ ${ }^{1}$ Волгоградский государственный университет, \\ 400062 Волгоград, Россия \\ ${ }^{2}$ Волгоградский институт бизнеса, \\ 400048 Волгоград, Россия \\ e-mail: yana_nn@volsu.ru
}

Поступила в редакцию 21.09.2018 г.

В окончательной редакции 19.11.2018 г.

Принята к публикации 20.11.2018 г.

Исследовано распространение двумерных предельно коротких оптических импульсов в графене с металлическими наночастицами. Изучены эффекты, наблюдаемые при изменении параметров эффективного гамильтониана, учитывающего обменное взаимодействие. Выявлено, что введение металлических адатомов в графен приводит к увеличению амплитуды импульса. Этот эффект можно усилить за счет выбора конкретной металлической частицы.

DOI: $10.21883 /$ OS.2019.03.47377.289-18

Уникальные электрофизические свойства графеновых структур открывают широкие возможности их практического использования в полупроводниковой технике. В связи с этим достаточно актуальным является вопрос, связанный с исследованием особенностей распространения электромагнитных импульсов в данных структурах с учетом композиции с металлическими наночастицами $(\mathrm{Pt}, \mathrm{Au}, \mathrm{Ag}$ и др.), которые способны существенным образом менять спектр базового материала (графен) [1] и улучшать его свойства [2-5]. Это и определяет основную цель настоящей работы.

К настоящему времени исследованы закономерности распространения предельно коротких оптических импульсов (ПКОИ) в системе полупроводниковых и металлических углеродных нанотрубок, в том числе с учетом воздействия внешних полей и неоднородности поля по их длине [6,7]. Была подтверждена экспериментом возможность генерации фемтосекундных импульсов в массиве УНТ [8]. Показана возможность усиления данных импульсов в графене под действием внешнего поля [9]. При этом без внимания остается вопрос влияния металлических наночастиц на эволюцию предельно коротких импульсов в графеновых структурах.

Проведение изучения композитов графена с металлическими наночастицами является важной задачей, поскольку их положительное влияние на свойства графеновых структур уже продемонстрировано во многих работах, относящихся к различным прикладным областям $[10,11]$.

\section{Основные уравнения}

Получим выражение для электронного энергетического спектра графена с учетом наличия примесей. В слу- чае адсорбированных наночастиц электронные свойства графена могут быть описаны с помощью эффективного гамильтониана, который выбирается в виде $[12,13]$

$$
H=H_{0}+H_{\Delta}+H_{i n t}+H_{R},
$$

где $H_{0}$ описывает кинетическую энергию носителей заряда; $H_{\Delta}$ описывает потенциальную энергию; обменные эффекты определяются гамильтонианом $H_{\text {int }}$; взаимодействие Рашбы описывается с помощью четвертого слагаемого в формуле (1) $-H_{R}$.

Явный вид каждого из четырех гамильтонианов приводится в формуле (2):

$$
\begin{gathered}
H_{0}=\hbar v_{D}\left(\tau k_{x} \sigma_{x}+k_{y} \sigma_{y}\right), \\
H_{\Delta}=\Delta \sigma_{z} s_{0}, \\
H_{i n t}=\lambda_{A} \frac{\sigma_{z}+\sigma_{0}}{2} s_{z}+\lambda_{B} \frac{\sigma_{z}-\sigma_{0}}{2} s_{z}, \\
H_{R}=\lambda_{R}\left(\tau \sigma_{x} s_{y}-\sigma_{y} s_{x}\right) .
\end{gathered}
$$

Здесь $k_{x}, k_{y}$ - компоненты волновой функции носителей заряда, $v_{D}-$ скорость электронов в окрестности дираковской точки, $\tau= \pm 1$ (соответствует точкам Дирака $K$ и $\left.K^{\prime}\right), \sigma_{x}, \sigma_{y}, \sigma_{z}, s_{x}, s_{y}, s_{z}$ - матрицы Паули в спиновом пространстве и пространстве решеток, $\Delta-$ полуширина запрещенной зоны, $s_{0}$ - единичная матрица в спиновом пространстве, $\lambda_{A}, \lambda_{B}-$ константы обменного взаимодействия для двух подрешеток графена, $\lambda_{R}$ - константа взаимодействия Рашбы.

Значения параметров гамильтониана $H$ в конфигурации над узлами решетки графена приведены в таблице. 
Значения параметров гамильтониана [14]

\begin{tabular}{c|c|c|c|c}
\hline Адатом & $\Delta$ & $\lambda_{A}$ & $\lambda_{B}$ & $\lambda_{R}$ \\
\hline $\mathrm{Cu}$ & 7.885 & 1.973 & 0.159 & 1.6 \\
$\mathrm{Ni}$ & 29.534 & 60.820 & 8.907 & 4.9
\end{tabular}

Перепишем гамильтониан (1) в матричной форме, вводя следующие обозначения:

$$
H=\left(\begin{array}{cccc}
\alpha & 0 & a & * b \\
0 & \beta & c & a \\
a^{*} & c^{*} & \gamma & 0 \\
b^{*} & a^{*} & 0 & \delta
\end{array}\right), \quad \alpha=\Delta+\lambda_{A}, \quad \beta=\Delta-\lambda_{A},
$$

$$
a=\hbar v_{D}\left(\tau k_{x}-i k_{y}\right), \quad b=i \lambda_{R}(1-\tau), \quad c=i \lambda_{R}(1+\tau)
$$

Решая далее задачу на собственные значения, получаeM

$$
\begin{gathered}
\varepsilon^{4}+A \varepsilon^{2}+B \varepsilon^{2}+C \varepsilon+D=0 \\
A=-(\alpha+\beta+\gamma+\delta)=0 \\
B=\alpha \beta+(\alpha+\beta)(\gamma+\delta)+\gamma \delta-2|a|^{2}-|b|^{2}-|c|^{2} \\
C=-\alpha \beta(\gamma+\delta)-\gamma \delta(\alpha+\beta)+|c|^{2}(\alpha+\beta)+|b|^{2}(\beta+\gamma), \\
D=\alpha \beta \gamma \delta-\alpha \gamma|a|^{2}-\alpha \delta|c|^{2}+|a|^{4}-a a b^{*} c^{*} \\
-|a|^{2} \beta \delta-|b|^{2} \beta \gamma-a^{*} a^{*} b c+|b|^{2}|c|^{2}
\end{gathered}
$$

Уравнение (4) будем решать с помощью метода Декарта-Эйлера [15], который позволяет свести уравнение 4-й степени к кубическому

$$
\begin{gathered}
w^{4}+0.5 p w^{2}+\frac{p^{2}-4 r}{16} w-\frac{q^{2}}{64}=0, \\
p=-\frac{3 A^{2}}{8}+B, \quad q=\frac{A^{3}}{8}-\frac{A B}{2}+C, \\
r=-\frac{3 A^{4}}{256}+\frac{A^{2} B}{16}-\frac{A C}{4}+D .
\end{gathered}
$$

В этом случае несложно получить выражение для электронного энергетического спектра $\varepsilon_{i}$ (корни уравнения (4))

$$
\begin{gathered}
\varepsilon_{1}=\sqrt{z_{1}}+\sqrt{z_{2}}+\sqrt{z_{3}}, \quad \varepsilon_{2}=\sqrt{z_{1}}-\sqrt{z_{2}}+\sqrt{z_{3}}, \\
\varepsilon_{3}=\sqrt{z_{1}}-\sqrt{z_{2}}-\sqrt{z_{3}}, \quad \varepsilon_{4}=\sqrt{z_{1}}+\sqrt{z_{2}}-\sqrt{z_{3}}, \\
z_{1}=w_{i}-\frac{p}{6}, \quad w_{1}=A_{1}+B_{1}, \\
w_{2,3}=-\frac{A_{1}+B_{1}}{2} \pm i \frac{A_{1}-B_{1}}{2}, \\
A_{1}=\left(0.5 q_{q}+\sqrt{Q_{1}}\right)^{1 / 3}, \quad B_{1}=\left(-0.5 q_{1}-\sqrt{Q_{1}}\right)^{1 / 3}, \\
Q_{1}=\left(\frac{p_{1}}{3}\right)^{3}+\left(\frac{q_{1}}{2}\right)^{2}, \\
p_{1}=-\frac{p^{2}}{12}+\frac{p^{2}-4 r}{16}, \quad q_{1}=\frac{p^{3}}{108}-\frac{p}{6} \frac{p^{2}-4 r}{16}-\frac{q^{2}}{64}
\end{gathered}
$$

Уравнение, описывающее распространение двумерного предельно короткого импульса, можно записать в следующем виде:

$$
\frac{\partial^{2} A}{\partial x^{2}}+\frac{\partial^{2} A}{\partial z^{2}}-\frac{1}{c^{2}} \frac{\partial^{2} A}{\partial t^{2}}+\frac{4 \pi}{c} j=0
$$

где $A-$ вектор-потенциал, с - скорость света, $j$ электрический ток.

В низкотемпературном пределе ток можно определить согласно формуле

$$
j=e \int_{-\Delta}^{\Delta} \int_{-\Delta}^{\Delta} d p_{x} d p_{y} v_{y}\left(p-\frac{e}{c} A(, x, z, t)\right) .
$$

Здесь $v_{y}\left(p_{x}, p_{y}\right)=\frac{\partial \varepsilon\left(p_{x}, p_{y}\right)}{\partial p_{y}}-$ скорость электронов.

Область интегрирования по импульсам можно определить из условия равенства числа частиц:

$$
\int_{-\Delta}^{\Delta} \int_{-\Delta}^{\Delta} d p_{x} d p_{y}=\iint_{Z B} d p_{x} d p_{y}\left\langle a_{q x, q y}^{+}, a_{q x, q y}\right\rangle,
$$

где интегрирование справа проводится по первой зоне Бриллюэна.

\section{Результаты численного моделирования}

Исследуемое уравнение (7) решалось численно [16]. Начальное условие в двумерном случае выбиралось в виде предельно короткого импульса, состоящего из одного колебания поля и распространяющегося вдоль оси $z$ :

$$
\begin{array}{r}
A(x, z, 0)=Q \exp \left(-\frac{z^{2}}{\gamma_{z}}\right) \exp \left(-\frac{x^{2}}{\gamma_{x}}\right), \\
\frac{d A(x, z, 0)}{d t}=\frac{2 z v_{z}}{\gamma_{z}} Q \exp \left(-\frac{z^{2}}{\gamma_{z}}\right) \exp \left(-\frac{x^{2}}{\gamma_{x}}\right) .
\end{array}
$$

Здесь $Q$ - амплитуда импульса, $v_{z}$ - его начальная скорость в направлении $z, \gamma_{z}, \gamma_{x}$ - определяют ширину импульса.

Возникающая эволюция двумерного электромагнитного поля при его распространении по графеновому образцу с наночастицами меди представлена на рис. 1.

Как видно, импульс распространяется достаточно стабильно, сохраняя свою форму. При этом стоить отметить образование „хвоста“, следующего за основным импульсом.

Влияние металлических наночастиц на характер распространения предельно короткого оптического импульса показано на рис. 2.

Рисунок 2 показывает, что введение металлических наночастиц оказывает достаточно сильное влияние на форму электромагнитного импульса. Поскольку, переходя к размерным единицам, получаем разницу в амплитуде порядка $0.1 \mathrm{MV} / \mathrm{m}$. При этом введение наночастиц 


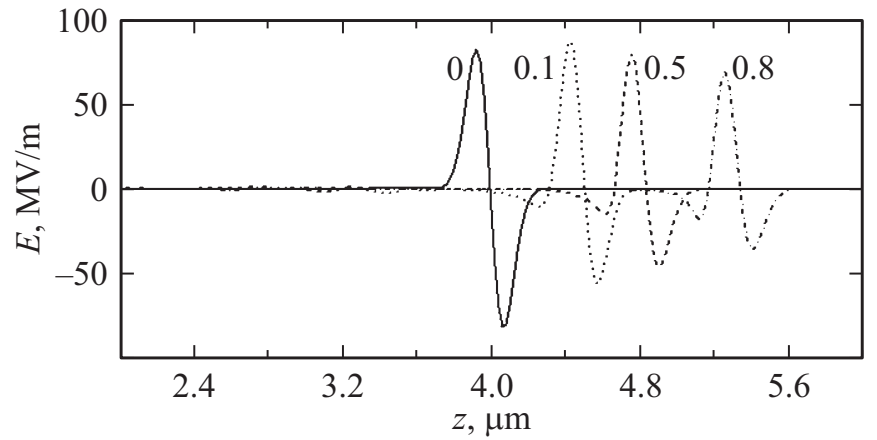

Pис. 1. Напряженность электрического поля двумерного электромагнитного импульса с металлическими наночастицами $\mathrm{Cu}(t)$ в моменты времени $t=0,0.1,0.5,0.8 \mathrm{ps}$.

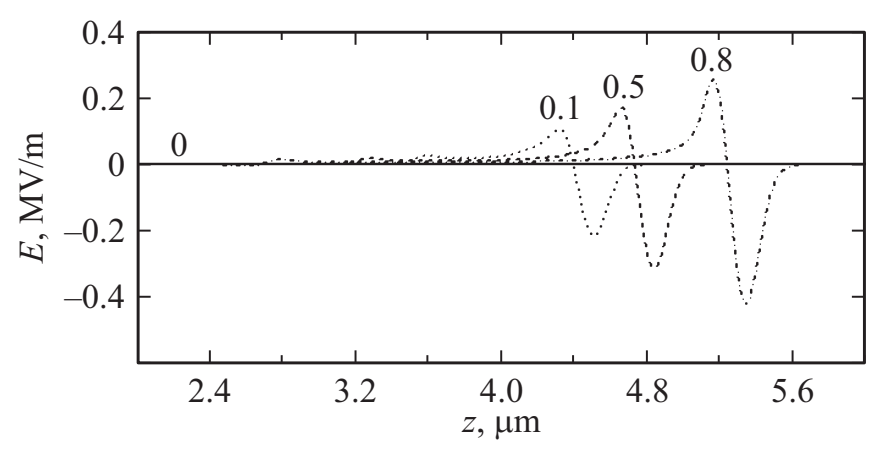

Рис. 2. Разность напряженностей электрического поля двумерного электромагнитного импульса с металлическими наночастицами $\mathrm{Ni}(t)$ и без них в моменты времени $t=0,0.1,0.5,0.8 \mathrm{ps}$.

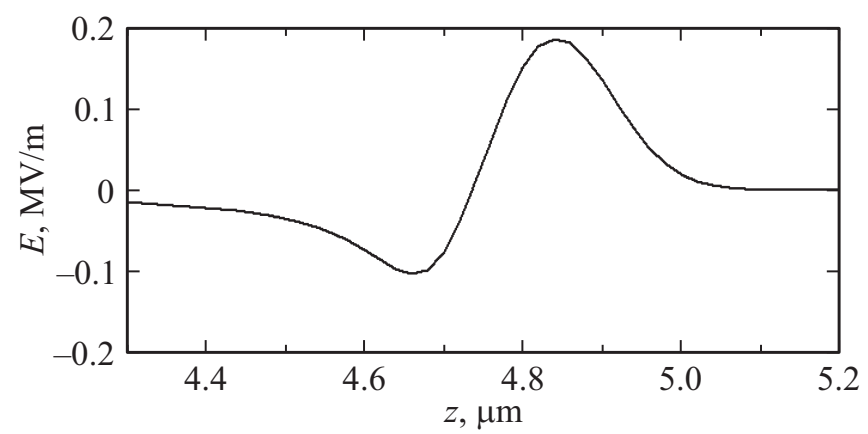

Рис. 3. Разность напряженностей электрического поля двумерного электромагнитного импульса для адатома $\mathrm{Ni}(t)$ и $\mathrm{Cu}(t)$ в момент времени $t=0.5 \mathrm{ps}$.

с течением времени приводит к усилению импульса по сравнению с беспримесным случаем.

Влияние типа адатома продемонстрировано на рис. 3 . Для наглядности на рис. 3 изображены не сами напряженности электрического поля в случае двух адатомов, а их разности. Как видно, тип металлической наночастицы адсорбированной на графене оказывает существенное влияние на амплитуду импульса, которое обусловлено изменением ширины запрещенной зоны, обменного вза- имодействия для двух подрешеток графена и взаимодействия Рашбы.

\section{Заключение}

1. Изучено влияние металлических частиц в графене на характер распространения предельно коротких оптических импульсов в данной структуре.

2. Показано, что увеличение амплитуды импульса наблюдается при введении в графен металлических адатомов. Этот эффект можно усилить за счет выбоpa конкретной металлической частицы. Так, для $\mathrm{Ni}(t)$ амплитуда увеличивается на $0.5 \%$, что соответствует величине $0.1 \mathrm{MV} / \mathrm{m}$.

3. Графен с металлическими наночастицами может найти широкое применение в области нелинейной оптики, например, в качестве нелинейного оптического элемента для ультрабыстрых волноводных лазеров.

Работа поддержана Российским фондом фундаментальных исследований (проект № 16-07-01265-А). Численное моделирование с использованием параллельных технологий выполнено при финансовой поддержке Минобрнауки РФ в рамках государственного задания (проект № 2.852.2017/4.6).

\section{Список литературы}

[1] Novoselov K.S., Geim A.K., Morozov S.V., Jiang D., Zhang Y., Dubonos S.V., Grigorieva I.V., Firsov A.A. // Science. 2004. V. 306, N 5696. P. 666. doi 10.1126/science. 1102896

[2] Kim I.-D., Choi S.-J., Cho H.-J. Graphene-Based Composite Materials for Chemical Sensor Application. Electrospinning for High Performance Sensors, Springer International Publishing, Cham, 2015. 65-101 p.

[3] Shang L., Bian T., Zhang B., Zhang D., Wu L.Z., Tung C.H., Yin Y, Zhang T. // Angew. Chem. 2014. V. 53. P. 250. doi 10.1002/anie.201306863

[4] Huang J., Zhang L., Chen B., Ji N., Chen F., Zhang Y., Zhang Z. // Nanoscale. 2010. V. 2. P. 2733. doi 10.1039/c0nr00473a

[5] Tan L.-L., Ong W.-J., Chai S.-P., Mohamed A.R. // Appl. Catal. B: Environ. 2015. V. 166. P. 251. doi 10.1016/j.apcatb.2014.11.035

[6] Конобеева Н.Н., Белоненко М.Б. // ФТТ. 2013. Т. 55. Вып. 10. С. 2008.

[7] Федоров Э.Г., Конобеева Н.Н., Белоненко М.Б. // ФТТ. 2014. T. 48. B. 10. C. 1383.

[8] Keiu K., Jones R.J., Peyghambarian N. // IEEE Photonics Technology Letters. 2010. V. 22 (20). P. 1521. doi 10.1109/LPT.2010.2063423

[9] Янюшкина Н.Н., Белоненко М.Б., Лебедев Н.Г. // Опт. и спектр. 2010. Т. 108. № 4. С. 658.

[10] Subrahmanyam K.S., Manna A.K., Pati S.K., Rao C.N.R. // Chem. Phys.Lett. 2010. V. 497 N (1-3). P. 70. doi 10.1016/j.cplett.2010.07.091

[11] Gutés A., Hsia B., Sussman A., Mickelson W., Zettl A., Carraroa C., Maboudian R. // Nanoscale. 2011. V. 4. N 2. P. 438. doi 10.1039/C1NR11537E 
[12] Zollner K., Gmitra M., Frank T., Fabian J. // Phys. Rev. B. 2016. V. 94. P. 155441. doi 10.1103/PhysRevB.94.155441

[13] Dyrda A., Barna's J. // 2D Materials. 2017. V. 4. P. 034003. doi 10.1088/2053-1583/aa7bac

[14] Wawrzyniak-Adamczewska M. Electronic and magnetic properties of the graphene densely decorated with $3 \mathrm{~d}$ metallic adatoms // arXiv:1711.09828 (2017)

[15] Korn G.A., Korn T.M. Mathematical Handbook for Scientists and Engineers: definitions, theorems, and formulas for reference and review. General Publishing Company, London, 2000. 1151 p.

[16] Thomas J.W. Numerical partial differential equations-finite difference methods. NY: Springer-Verlag, 1995. 437 p. 